\title{
LA ORGANIZACIÓN DEL CONTENIDO DE ENSEÑANZA EN CARRERAS UNIVERSITARIAS.
}

\section{THE ORGANIZATION OF THE TEACHING CONTENTS IN UNIVERSITY CAREERS}

\author{
MC. José Manuel Ruiz Socarras \\ jruiz@inf.reduc.edu.cu \\ Dr. Gaspar Barreto Argilagos\% ${ }^{* *}$ \\ mago@con.reduc.edu.cu \\ Dr. Ramón Blanco Sánchez ${ }^{\text {***\% }}$ \\ ramblan@inf.reduc.edu.cu
}

\section{Resumen}

El propósito del trabajo fue fundamentar la organización del contenido de enseñanza en carreras universitarias como un problema científico aun sin resolver completamente. Se empleó fundamentalmente el método del enfoque sistémico. Se caracterizó el proceso de organización y se estudió su comportamiento en diferentes modelos curriculares y por diferentes autores, haciendo hincapié en la utilización de métodos de la teoría de grafos en la organización del contenido, llegándose a la conclusión de que se requiere una organización del contenido-enseñanza que permita un funcionamiento adecuado del plan de estudio como sistema.

Palabras clave: organización, contenido, enseñanza, diseño curricular, teoría de grafos.

\section{Abstract}

Give reasons about the organization of the teaching contents in university careers as a scientific problem not solved yet in its totality has been the subject of this work. The systemic focus was the method used in general. The process of organization was characterized and its behavior through

* Universidad de Camagüey. Cuba. Facultad de Informática. Departamento de Matemática.

** Universidad de Camagüey. Cuba. Facultad de Construcciones. Departamento de Ingeniería Civil.

**** Universidad de Camagüey. Cuba. Facultad de Informática. Departamento de Matemática. 
different curricular models and by several authors was analyzed, pointing up the uses of Net Theory about content organization, arriving to the conclusion that an organization of the teaching content is required to allow an adequate working of the scholastic curriculum as a system.

Key words: organization, content, teaching, curricular model, net theory.

\section{LA ORGANIZACIÓN DEL CONTENIDO DE ENSEÑANZA}

Organizar es "dar a las partes de un todo la organización necesaria para que puedan funcionar", "disponer las partes de un todo de manera que puedan funcionar", mientras que la palabra organización significa "acción y efecto de organizar u organizarse. Disposición, arreglo, orden", y la palabra orden significa, "colocación de las cosas en el lugar que les corresponde. Concierto, buena disposición de las cosas entre sí (o de las partes que constituyen un todo). Serie o sucesión de las cosas. Relación o respecto de una cosa a otra".

Como se aprecia en las definiciones anteriores, la acción de organizar recae sobre las partes de un todo para que puedan funcionar. Aparecen las categorías del todo y las partes; de ahí que, entre otros aspectos, los autores del artículo consideran apropiado abordar la organización del contenido de enseñanza como un sistema.
Diversos son los autores que reconocen la necesidad e importancia de organizar el contenido de enseñanza. Según Gimeno (1996: 358) una cuidadosa selección y ordenación pedagógica del contenido es de vital importancia para los profesores y es una función capital del diseño curricular. Señala que, en muchos casos, la intervención de los profesores en esa operación se ve dificultada por la carencia de la capacitación técnico-pedagógica y cultural suficiente y adecuada.

Loredo (2003: 11) cita a Bobbitt, Tyler y Taba, quienes consideran necesario introducir una secuencia lógica y racional en la enseñanza, fijar claramente los objetivos y los contenidos que deben ser aprendidos, las experiencias que deben ser realizadas, establecer la apropiada organización y secuencialización de contenidos y actividades, así como la forma de evaluar el logro de los objetivos.

Orden. En: Diccionario Enciclopédico SALVAT Universal., t. XVI, 1973.-p.540. 
Coll, citado por Gimeno (1996: 360), señala que situándonos dentro de una disciplina, en un área o en la opción de relacionar áreas y disciplinas diversas, la forma de presentar organizados y agrupados los contenidos tiene enorme importancia, por cuanto la decisión que se tome condiciona también las relaciones posibles que pueda establecer el alumno en su aprendizaje. Gimeno plantea que el profesor debe cuestionarse la mejor forma de organizar el contenido del currículo. Que la secuencia de unidades ofrece alternativas diversas por concretar de acuerdo con el contenido y las opciones pedagógicas, lo cual resulta un problema difícil de resolver.

Briggs (1973), citado por Gimeno (1996: 361), señala que la estructura del conocimiento a tener en cuenta cuando pretendemos que se aprenda supone establecer unas relaciones de dependencia y de independencia de las partes integrantes, dispuestas de modo que indiquen cuándo puede ser optativo o aleatorio el orden de la secuencia y cuándo ha de hacerse cuidadosamente para lograr una trayectoria óptima para alcanzar destrezas u objetivos complejos.

Díaz (1994: 8) señala que la construcción de objetivos en términos de comportamiento ha sido un elemento básico y privilegiado del desarrollo del campo del currículo, y que esto dejó en un plano olvidado el problema de los contenidos, de manera que las investigaciones en relación éstos, tienen poco significado en el ámbito curricular. Plantea que las técnicas para el tratamiento del contenido son insuficientes y no tan claras ni tan específicas como las que existen para formular objetivos, pero que el tratamiento de los contenidos tiene un factor de innovación mucho más fuerte que los objetivos, máxime cuando se observa que la investigación sobre el problema de los contenidos desemboca en dos temas básicos para el trabajo en el aula: los procesos de aprendizaje y las estrategias metodológicas de enseñanza. En resumen, aprecia una pérdida del papel de la investigación para fundamentar decisiones y un claro detrimento de los estudios respecto del contenido, al haber constituido como eje de los planes y programas el problema de los objetivos. Él da gran valor al papel de la investigación en la toma de decisiones, en la construcción de planes de estudio y su organización, resultado de un proceso de indagación. Esta cuestión, que desde los inicios del desarrollo de la teoría curricular, fue fundamentada y permanentemente tratada en los desarrollos por diferentes autores como Tyler, Taba, Díaz Barriga, pero se ha perdido.

Diversos autores como Álvarez (1995), citada por Añorga (1997: 22), Loredo (2003: 12), Posner, citado por Enríquez (2003: 36), Darós (1993: 42), señalan diversas fuentes o dimensiones que se deben tener en cuenta para el diseño curricular de planes, programas de estudio y cualquier otro elemento que sea parte del curriculo. Sin embargo, de una u otra forma, reconocen la necesidad de la selección de objetivos y contenidos, el establecimiento de secuencias lógicas y científicas de aprendizaje que favorezcan al máximo la asimilación 
de los contenidos y del logro de los objetivos, la importancia de la organización del contenido.

Para Tyler (1986: 86), la organización es un problema importante de la formulación del curriículo ya que influye muchísimo en la eficacia de la enseñanza.

Si se analizan los diferentes grupos de metodologías de diseño curricular, se encuentra que aunque responden a diferentes modelos curriculares $^{1}$, todas se enfocan en la organización y secuenciación de contenidos de una u otra forma.

Así pues, dentro de las metodologías curriculares clásicas, autores como D’Hainaut, Le Xuan y Morganov, estudiaron la organización y secuenciación de contenidos y aportaron técnicas para analizarlas.

Entre las metodologías curriculares cuyo enfoque es crítico y sociopolíti$\mathrm{co}$, se destaca la Propuesta Curricular Modular por objetos de transformación, cuya metodología marcó un hito en la concepción y práctica educativa de las instituciones de educación superior en los años de 1970, y es el resultado de una experiencia en la elaboración de planes de estudio por Ángel Díaz Barriga en la Universidad Autónoma Metropolitana de México. Una de las características es que, en oposición al diseño curricular por asignatura, aboga por la utilización de módulos por objetos de transformación, consistentes en la integración del contenido a partir de un problema de la realidad, que se toma como tal, en su totalidad y como proceso, para explicarlo por la vía de la acción sobre él, mediante un estudio interdisciplinar. A partir de esto se planteó organizar el currículum. Es la resolución de un problema la que exige, en determinado momento, el ordenamiento de cierto tipo de información. Esta información no procede de la lógica de la disciplina ni respeta los límites formales establecidos para cada asignatura.

En relación con la metodología curricular constructivista, se tiene como representativo en ella el trabajo de César Coll (1987-1990), quien dirigió el proceso de reforma curricular de la Educación Básica Española. Aunque está dirigida a la enseñanza obligatoria y no a la Superior, a partir de un modelo de diseño curricular base, el autor considera importante los niveles o fases que establece:

1. Se especifican los objetivos generales, las áreas curriculares pertinentes, los bloques de contenidos y orientaciones didácticas.

2. Se realiza el análisis y secuenciación de los bloques de contenido.

3. Se desarrollan ejemplos de programación atendiendo a los diversos supuestos establecidos. 
Las metodologías curriculares constructivistas centran la atención en cómo aprende el alumno, considerando el aprendizaje como un proceso constructivo.

La teoría de Ausubel (1989) del aprendizaje significativo se fundamenta en el constructivismo como plataforma para aprender significativamente, en la que los conocimientos previos son los fundamentos de la construcción de los nuevos significados. Al respecto Voss (1978), citado por Lara (1997: 35) señala que lo más importante para aprender algo no es lo que se va a aprender, sino lo ya aprendido, porque es con lo que tiene que relacionarse para que adquiera significado.

Según Lara (1997: 43), en general, los alumnos tienen dificultades para vincular la nueva información con los conocimientos previos, cuando no se lo proponen, cuando el alumno no se encuentra en una disposición o actitud favorable para aprender, cuando no está motivado o interesado para realizar un esfuerzo intencionado en relación con los contenidos de aprendizaje o cuando la información de que dispone es poco clara, está desorganizada o de alguna forma carece de sentido. La existencia de conocimientos previos no es suficiente, lo fundamental es que los utilicen en el momento adecuado para establecer relaciones con el nuevo contenido; para lograrlo, los alumnos deben ser capaces de tener acceso a lo que saben y vincular la información que reciben con otra información a través de estrategias de recuperación.
Según Novak, citado por González y Reinoso (2002: 186) una de las interrogantes interesantes que la teoría del aprendizaje significativo de Ausubel trata de responder es cómo se deben organizar los contenidos curriculares.

En la metodología histórico-cultural, el contenido de la enseñanza se agrupa por unidades coherentes, siendo la agrupación más tradicional la de disciplina académica, aunque en la actualidad, en las condiciones de trabajo profesional, esta agrupación es más multidisciplinaria, lo cual garantiza enfoques más integrales y productivos para las tareas profesionales. Aquí también se determina la secuencia de impartición de los contenidos, que se enfocan a partir de la relación dialéctica entre objetivo y contenido.

Por una parte, para el autor, el hecho de que el contenido de enseñanza se nutre del objeto de la cultura, el que está integrado por conocimientos, lógica y métodos de sus diferentes ramas (ciencia, tecnología, arte, tradiciones, creencias) hace que dicho contenido tenga una naturaleza compleja, ya que compleja es la estructura del objeto de la cultura, que es amplia y diversa en sus partes componentes y en las relaciones que entre ellas se establecen.

Por tanto, si se acepta que el contenido de enseñanza es complejo, también debe ser complejo su proceso de organización, aunque no solo por tal motivo, sino por el propio carácter contradictorio de los requerimientos de dicho proceso de organización, 
manifiestos en el tener que tomar una parte de la compleja y amplia estructura del objeto de la cultura, respetar su lógica y estructurarla de la forma más sencilla y asequible, de modo que el estudiante la asimile en el periodo poco extenso de tiempo de duración de la carrera y, con ello, consiga los objetivos propuestos como profesional.

Es decir, se trata de una estructura compleja y amplia, a partir de la cual se debe conformar una estructura que se asimile de forma sencilla en un periodo no excesivo. Por tanto, de la contradicción entre lo complejo del objeto de la cultura y lo sencillo que debe resultar la asimilación del contenido de enseñanza, por una parte, y de la contradicción entre lo extenso del objeto de la cultura y lo limitado del tiempo de duración de la carrera, por otra, es que emerge la complejidad del proceso de organización del contenido de enseñanza.

Si se acepta que el proceso de organización del contenido es un proceso complejo, no se puede aceptar que en un proceso de tal naturaleza, haya prevalencia de empirismo y sobrevaloración o predominio del criterio de expertos. Se impone la necesidad de que los expertos dispongan de instrumentos metodológicos adecuados que faciliten la organización del contenido sobre la base de un acertado desarrollo teórico.

A lo largo de la historia el contenido de enseñanza se ha organizado y se continúa organizando de diferentes formas. Unidades didácticas, proyectos de aprendizaje, núcleos interdisciplinares, módulos, áreas o disciplinas, asignaturas son denominacio- nes que, desde diversas ópticas, autores o movimientos pedagógicos, apelan al diseño curricular. Sin embargo, cualquiera sea la denominación que se utilice para organizar el contenido, ella es expresión de las partes componentes del contenido, pero estas partes se relacionan entre sí. Esto debe ser tenido en cuenta en el proceso de enseñanzaaprendizaje, ya que el principio de la sistematicidad de la enseñanza señala que, por la particularidad psicofisiológica del hombre, le es más fácil asimilar conocimientos estructurados (relacionados) que información fragmentaria, que no se puede estudiar en forma cabal un nuevo contenido de enseñanza si no se ha asimilado el contenido que lógicamente lo fundamenta y con el cual está relacionado de manera sistemática.

Tyler (1986: 102) reconocía las ventajas de una estructura que establezca periodos más amplios, que tienda a eliminar fronteras entre las asignaturas, en contraposición de unidades muy estrechas, al igual que la excesiva cantidad de asignaturas, en cuyos casos se dificulta elaborar la relación de cada una de ellas con las demás y en al que cada una requiere algún tipo de transición y consideración de la labor de las otras.

Aunque señala que esta dificultad aumenta a medida que aumenta el número de "piezas" o asignaturas, al mismo tiempo plantea los inconvenientes de una organización indiferenciada del día escolar, dado que los alumnos necesitan pasar constantemente de una actividad a otra para evitar la fatiga, que además les permite estar en contacto con más 
de un adulto, que puede también ser más competente.

Por tanto, la organización del contenido de enseñanza no es una tarea fácil, si se quieren garantizar las relaciones necesarias entre sus partes componentes, dada la diversidad del objeto de la cultura y el número de asignaturas que actualmente conforman los planes de estudios universitarios. Por el contrario, es muy compleja y por tanto requiere instrumentos metodológicos que la faciliten, fundamentados en un desarrollo teórico conveniente.

\section{Utilización de mÉtodos de la TeOría de Grafos en la ORganización del ConTENIDO dE ENSEÑANZA}

El problema fundamental no estriba en aceptar la necesidad o importancia de algo (es condición necesaria pero no suficiente), sino en cómo hacerlo efectivo, cómo llevarlo a la práctica. Esto plantea la necesidad de encontrar procedimientos viables y conceptualmente fundamentados para resolver el problema.

Un ejemplo de la importancia de la práctica en relación con la teoría puede encontrarse en la relación entre los mapas conceptuales como instrumento que puede facilitar un aprendizaje significativo-constructivista en la práctica. Ausubel no se preocupó por proporcionar a los educadores instrumentos simples y prácticos para ayudarles a averiguar lo que el alumno ya sabe, pero Novak (1988), trabajando con base en la teoría de Ausubel, diseñó uno de estos instrumentos que llamó mapas conceptuales, los cuales son una proyección práctica de la teoría del aprendizaje de Ausubel.

Salgado (1979: 78) señala:

Es de tal magnitud la tarea de revisión y redacción de los programas de materias y relaciones intermaterias de todas las asignaturas, grados, niveles y especialidades de los distintos subsistemas de educación, que el control y la seguridad de estos programas, realizados muchas veces con criterios nuevos y contenidos que se imparten por primera vez, se hace extremadamente difícil. Ni aun los especialistas mas calificados pueden asegurar la perfección de los programas revisados por ellos cuando el número de contenidos y relaciones es significativo.

El uso de métodos matemáticos que formalicen y permitan automatizar las partes más voluminosas del proceso de revisión y confección de programas (especialmente los de relaciones intermaterias) considerando todos los elementos pedagógicos, es producto de la necesidad de elevar la eficiencia y productividad de los colectivos que los preparan con un consiguiente incremento de la efectividad de la enseñanza.

Él propone un método en que define un programa como un grafo $\mathrm{G}(\mathrm{V}, \mathrm{U})$, 
donde $\mathrm{V}$ es el conjunto de contenidos de una o varias materias y $U$ las interrelaciones que entre ellas se establecen. A este grafo se asocia una matriz booleana en la que, mediante programas computacionales, se determina:

1. Detección de los "circuitos" (contradicciones lógicas entre los contenidos relacionados).

2. Detección de contradicciones en los momentos de introducción de contenidos intermaterias relacionadas, de acuerdo con los procedimientos establecidos.

3. Ordenación y optimización de los contenidos del programa de acuerdo con parámetros que reflejen los criterios pedagógicos planificados por los autores.

Aunque valoramos altamente el trabajo desarrollado por Salgado, pero sostienen la idea de que no es suficiente la detección de contradicciones lógicas entre los contenidos relacionados, sino que es necesario clasificarlas e indicar procedimientos lógicos para superarlas.

También consideramos que la experiencia cubana en la utilización de métodos de la teoría de grafos en la organización del contenido de enseñanza tiene sus antecedentes en la experiencia de avanzada en investigaciones pedagógicas de los países del antiguo campo socialista, la que se enmarca en las aplicaciones internacionales se los años 70 como técnicas de secuenciación de contenidos y análisis conceptual en materias de fuerte estructura lógica, en las que reconocen que ciertos conceptos son condición previa para el aprendizaje de otros y, con base en una matriz, determinan en qué orden deben presentarse determinados contenidos de aprendizaje; por ejemplo, el caso de la determinación del orden en que deben presentarse las operaciones aritméticas. Se recomienda ordenar los temas de acuerdo con su nivel de complejidad. Se debe usar el sistema Morganov-Heredia para organizar temas cuya complejidad requiera un sistema como éste.

Esta tendencia alcanzó su vigencia a mediados de los años 80 y fueron seriamente cuestionadas por la visión técnica y poco reflexiva con que se emplearon, y por las expectativas que se generaron con su uso, las cuales no fueron satisfechas. No obstante dejaron huella, y algunos autores trabajan el análisis de contenido y de conceptos de maneras que tienen cierta semejanza con dichos enfoques.

Siroezhin y Jristova (1975: 24) refieren la experiencia en la utilización de un modelo matemático especial y del algoritmo para el análisis y la elaboración del plan de preparación de especialistas en la rama de Cibernética Económica en el Instituto de Economía y Finanzas de Leningrado, el cual contaba con 51 asignaturas.

Ellos reconocen que la secuencia y el volumen de las asignaturas adecuadas para ser asimiladas por el estudiante, estructuradas con eficacia no dan lugar a errores que traen consigo complicaciones que menoscaban el resultado final. Plantean que la primera y fundamental condición que debe ser tenida en cuenta cuando se vaya a elaborar el plan docente es la secuenciación de asignatura 
a asignatura, así como el volumen y la correlación de sus contenidos. Considera que en su aspecto esquemático, el proceso de capacitación de especialistas puede compararse con la elaboración de artículos por el método de ensamblaje en cadena y utilizar el sistema de modelos y algoritmos estructurales para la organización de los trabajos de ensamblajes complejos. El problema que pretendían resolver era hallar un orden tal que, sin detrimento de las condiciones vedadas de continuidad, garantice la máxima uniformidad de la carga docente por intervalos y que la duración y prolongación de las operaciones se mantengan dentro de determinados límites.

El problema planteado lo resolvían a partir del análisis de relaciones lógicas entre asignaturas: relaciones de precedencias y relaciones de simultaneidad. Aplicando el Método del peso de posición, distribuían las asignaturas por etapas de estudio.

El segundo método usado por los referidos autores, que difería del método anterior, era el Método de dicotomía, para el que disponían de un programa de Algol-60, que utilizaron para elaborar el plan docente para una de las especialidades de ingeniería, al distribuir en forma óptima 75 asignaturas en seis etapas en menos de tres minutos, según refieren.

Una nueva concepción en la elaboración de instrumentos que propicien la distribución de las asignaturas en el plan del proceso docente de las carreras universitarias, a diferencia de los anteriores métodos, tiene que estar encaminada a disminuir la visión técnica y poco reflexiva que ha sido objeto de crítica, lo que debe traducirse en mayor flexibilidad en el plan del proceso docente que se diseñe, ofreciendo posibles variantes. Debe primar la idea de que el experto en diseño se auxilie de la herramienta computarizada, aunque esta no sustituya el papel del experto en la toma de decisiones.

Para Krasnov (1977: 236) el proceso docente en el Centro de Educación Superior (CES) es multifacético y complejo, y al planificarlo se tendrán en cuenta todos los vínculos funcionales entre disciplinas y las partes que la integran, utilizando los esquemas lógico-estructurales (ELE) y gráficos radicales. Estos esquemas no solo permiten establecer y proyectar el plan docente, sino también optimizar la estructura de los cursos y elaborar el programa de su estudio. Se construye la matriz de las interrelaciones entre disciplinas y se fracciona el material en temas para simplificar la estructura de los ELE.

Krasnov señala que el uso de las computadoras presenta grandes posibilidades para la planificación óptima del proceso docente y que una serie de CES de los antiguos países socialistas han confeccionado y dispuesto algoritmos tipo para dar solución a las siguientes tareas de planificación:

Confección de planes docentes.

Elaboración del plan-gráfico docente del estudiante para el semestre (incluidas las tareas de casa).

Cálculo de horas/año de la carga docente de la cátedra. 
- Confección del horario de actividades docentes y exámenes.

La República Democrática Alemana realizó una gran labor de optimización de la planificación docente usando los logros de la ciencia en esos momentos. En la República Socialista de Checoslovaquia se investigó el uso de la técnica computacional en la solución de una serie de aspectos de la planificación del proceso docente. La República Socialista de Rumania elaboró la metódica de la confección de los planes docentes, orientada en el sistema de criterios de las especializaciones. En la URSS se realizaron vastas investigaciones de los problemas de la determinación del contenido y la planificación del proceso docente-educativo.

Para Bobkov y Butaev (1980: 104), en la elaboración de los planes docentes tiene mucha importancia garantizar la ubicación lógica correcta de las disciplinas que se estudian. En la Escuela Técnica Superior N. E. Bauman de Moscú, se aplicó con éxito la metodología de los esquemas lógico-estructurales temporales. Esta metodología se utilizó con algunos pequeños cambios en otros CES. Partiendo del análisis del contenido de todas las disciplinas del plan docente, se construyen las matrices y los grafos que muestran la relación interna entre los cursos, acápites y temas fundamentales, que permiten establecer su relación en el tiempo. A la par con el empleo de esta metodología se trabajó en un algoritmo de solución de la tarea, dirigido a coordinar las disciplinas en la computadora digital electrónica. Utilizando el método de grafo y esta última, se logró garantizar la concordancia lógicamente justificada de las disciplinas en el plan docente en todas las tendencias fundamentales de la capacitación del ingeniero.

Dimova, Malamov y Chalykov (1981: 166) determinan la estructura del contenido de la enseñanza por los nexos o relaciones entre descriptores, y consideran estos últimos como las unidades metodológicas elementales del contenido de la enseñanza (hechos científicos, conceptos, leyes, hipótesis, teorías, métodos, tecnologías, problemas, fines, etc.). De esta forma se hace discreto el contenido de la enseñanza y posibilita representarlo visualmente en forma de grafo orientado: descriptor-vértice y nexo-arco.

También señalan que la práctica en la utilización de este método muestra que para conformar la estructura de un contenido de la enseñanza no se requiere clasificar los nexos detalladamente, ya que no es necesario tener en cuenta todos los nexos entre descriptores, pues basta que los especialistas de las distintas asignaturas (expertos), basándose en las consideraciones lógicas, señalen los nexos primarios que determinan las relaciones entre ellos. La complejidad para determinar los nexos es diferente en las distintas asignaturas, lo que está dado por el propio carácter de la disciplina.

Según ellos, aunque los descriptores de cada asignatura son la base para conformar correctamente, desde el punto de vista metodológico, la estructura del contenido de la ense- 
ñanza de la especialidad, el gran número de descriptores y nexos hace que prácticamente no pueda utilizarse este nivel para planificar el proceso docente, lo que obliga a agrupar los descriptores en unidades metodológicas mayores (temas) y a establecer nexos agregados entre ellos.

Ante la pregunta de cómo ubicar mejor las asignaturas en el semestre para planificar el proceso docente, los referidos autores señalan que la planificación de temas o de unidades implica la realización de innumerables y arduas operaciones, lo que lleva a la conveniencia de utilizar computadoras, pero plantean que resulta cómodo y conveniente planificar tmas o unidades y que no es acertado la planificación de asignaturas.

Peón (1982: 59) señala que la planificación del proceso docente es una tarea de primer orden que, por su contenido y forma, se hace cada día más compleja. Por tal razón se deben emplear medios mecanizados para ejecutarla.

Para Saveliev, citado por Peón (1982: 59):

También en la esfera de la planificación debemos buscar formas para el perfeccionamiento del proceso docente. En efecto, no es un secreto que del plazo depende la calidad del trabajo de una empresa concreta. El CES constituye una "empresa" complejísima en la cual se "producen" especialistas de alta calificación. La planificación de esta "producción" y todo el proceso se realiza hasta ahora con métodos tradicionales.
Saveliev, teniendo en cuenta algunos de los problemas principales que al respecto surgen, plantea la necesidad de detectar la continuidad del estudio de las disciplinas, lógicamente relacionadas entre sí.

Volviendo al caso de Cuba, entre los documentos rectores sobre criterios y metodologías para el trabajo científico que redactó el Ministerio de Educación (MINED) sobre el Perfeccionamiento en el Subsistema de la Educación General en el periodo 1975-81, está el titulado Método de utilización de matrices para el análisis y evaluación de programas y la relación intermateria de enseñanza. Uso de la computadora (57).

Estos documentos se fundamentaban en los principios de la pedagogía socialista, marxista-leninista, producto de la experiencia acumulada y el desarrollo alcanzado por dicha ciencia en el sistema socialista mundial que encabezaba la URSS.

Estas normas del trabajo científicometodológico, que promovió el MINED, fueron aplicados en los estudios de diagnóstico y de pronóstico del desarrollo del Sistema Nacional de Educación en Cuba en el curso 19721973, y sirvieron de base al Plan de Perfeccionamiento propuesto para el periodo 1975-198 bajo la tutoría científica de la asistencia técnica soviética.

En el documento a que se hace referencia se señala que, además de la evaluación por expertos, cada programa puede ser analizado con la ayuda de matrices, lo cual permite establecer los vínculos entre los conceptos de una o varias asignaturas, descubrir los circuitos lógicos en la 
definición de algunos de ellos y aclarar la racionalidad del orden al introducir los conceptos y determinar aquellos que resulten fundamentales para el sistema conceptual de la ciencia.

También una de las ventajas del análisis por matrices está en la posibilidad de usar computadoras. La elaboración de un solo programa para la máquina que al ser elaborado de manera general permite trabajar sobre diversos contenidos de carácter concreto. Generalmente, las matrices se utilizan para establecer las relaciones entre algunos elementos; en este caso se les llama matrices de la red lógica de relaciones.

En Cuba el Instituto Técnico Militar usaba matrices y ELE con mucho cuidado en la elaboración de sus planes y programas de estudio.

A partir de la formulación de todas las materias del plan de estudio, éstas se agrupan en correspondencia con su precedencia, para lo cual se formula la "matriz de precedencias" y se van efectuando cortes, creándose grupos de materias que indican su secuencia lógica de estudio. Durante este proceso de corte surgen ciclos cuya solución está en el reagrupamiento de asignaturas o en la redistribución de su contenido entre otras afines.

La distribución de las asignaturas por grupos permite elaborar una propuesta de distribución por año de estudio, e incluso por semestre. El ELE es una distribución gráfica por años y semestres que indica la secuencia de su impartición tomando en cuenta sus relaciones.
Ellos señalan que el emplear la anterior secuencia en la confección de los planes y programas de estudio les permite:

Disminuir el número de asignaturas, haciendo más racionales los planes de estudio.

Erradicar los problemas de precedencias.

Esclarecier el papel que realmente corresponde a cada asignatura.

- Eliminar las duplicidades en cuanto a la información docente que brindan las asignaturas.

Lamas (1984: 74) utiliza matrices para analizar la articulación horizontal entre los programas de dos asignaturas que se imparten en un mismo semestre del plan de estudio vigente, determinando las relaciones contradictorias y, a partir de ellas, reestructurar el orden de las clases de ambas asignaturas, precisando en qué semanas deben empezar una $u$ otra asignatura, en caso de que no puedan empezar simultáneamente, aunque sí en el mismo semestre. Así se eliminan contradicciones entre ambas asignaturas y a la vez, se interrelacionan. A esta forma de utilización de las matrices la llaman Método Matricial.

Para Tristá (1985: 63), una de las técnicas mas utilizadas para la optimización de los planes y programas de estudio es la modelación en redes. Se diseña primero una red para los contenidos de cada una de las asignaturas y luego se estructura el modelo en red para el plan de estudio. Los gráficos de red pueden mostrar 
la estructura del plan de estudios y de los programas de asignaturas, la correlación entre todos sus elementos y el lugar que ocupa cada una de ellas en el sistema de enseñanza. También ayudan a excluir las duplicidades o a suprimir las cuestiones secundarias, a distribuir el material entre los cursos y coordinar los periodos de su estudio y hacer concordar el volumen de información con el tiempo disponible del estudiante.

Rodríguez (1985: 97) utiliza el Método Matricial para el análisis de los sistemas de relaciones existentes entre los conceptos de una asignatura, reconociendo que la lógica interna de las ciencias es el fundamento metodológico principal para elaborar los programas de cada asignatura en forma de sistema. Señala además que, en el caso de las disciplinas docentes, es necesario conformar la red lógica de conceptos entre todas las asignaturas, a través de la cual se pueden conocer la ubicación de la asignatura en el plan de estudio. Así mismo, a la relación que existe entre el concepto de grafo y el sistema de relaciones entre los conceptos.

Es necesario relacionar este enfoque del llamado Método Matricial con un concepto posterior, los mapas conceptuales (Novak, 1988), y relacionar ambos con la Teoría de Grafos, que en el caso de los mapas conceptuales es reconocida por Zapata (2001) en pleno siglo XXI.

Esta relación la manifiesta el Buró de Diseño Curricular de la Universidad de Camagüey ${ }^{2}$, cuando señala, entre los diferentes procedimientos para el análisis de los conceptos en cualquier tipo de currículo:

1. El método matricial.

2. La teoría de grafos.

3. Mapas conceptuales o curriculares.

Reconociendo diferentes usos en la Educación de los mapas curriculares:

1. como herramienta de enseñanza

a) Como recurso didáctico para la ayuda en el estudio basado en la teoría de Ausubel.

b) En la resolución de problemas, dado el papel esencial que juegan los conceptos en este tema. Aunque la formulación de conceptos y la solución de problemas fueron campos de investigación diferentes, hoy no lo son pues no se puede hablar de solución de problemas sin una base conceptual.

2. Como herramienta para estructurar una disciplina o parte de la misma, para estructurar programas y planes de estudio en general, siempre que se parta de un estudio de los objetivos.

Es de destacar el uso del Método Matricial, como herramienta matemática y de los mapas conceptuales, en el diseño curricular dirigido por este Buró. A finales de los años 90 el Buró propone una metodología para el estudio curricular y perfeccionamiento 
de las asignaturas y disciplinas, en la que el autor reconoce de positivo, por una parte, el cambiar el nombre de mapas conceptuales por el de mapas de contenido, para no caer en el error de limitar el análisis del contenido solo a los conceptos y olvidar las habilidades, $y$, por otra parte, valora de positivo el ordenar el plan de estudio según las precedencias de contenido, pero al mismo tiempo considera negativo que no ofrecen una teoría para realizar tal ordenamiento ni una metodología o instrumento que permita realizarlo.

La metodología que propone, no se proyecta para realizar el diseño curricular, sino el rediseño en un proceso de perfeccionamiento. Esto la limita en opinión del autor, aunque de hecho puede ser aplicable al diseño en sus orígenes.

Otra dificultad que aprecia el autor es que se absolutiza el papel de los objetivos en el proceso de enseñanza aprendizaje, por cuanto solo se reconoce la relación directa entre objetivo-contenido, pero no da lugar a la relación inversa contenido-objetivo en los diferentes niveles de sistematización de la carrera, lo que puede variar el tiempo de duración de la carrera, la estructura de semestre 0 los objetivos anuales.

Para el autor también es una limitante ubicar la determinación de un método de diseño de disciplinas y asignaturas posterior al ordenamiento, según precedencias del contenido del plan de estudio, ya que dicho método forma parte de la organización del contenido y, por tanto, está sometido a la relación que hay entre las categorías objetivo y contenido.

La organización del contenido de enseñanza ha estado marcada, en opinión del autor, por la opción de diseño curricular que se asuma frente a la contradicción entre profesionalización y fundamentalización.

Gutiérrez ${ }^{3}$ considera que, ante este problema, es necesario estructurar el contenido de la enseñanza a partir de componentes que expresen tanto la profesionalización como la fundamentalización. Por tanto, esos componentes tienen que estar en componentes del contenido de la ciencia asociados al modo de actuar del profesional; por ejemplo, los métodos, las habilidades, las tareas, y no en los conocimientos, pues éstos pertenecen sólo al contenido de la ciencia y acentúan la contradicción. Dichos componentes no pierden la fundamentalización propia de la ciencia y a la vez pueden ser tomados para la profesionalización. De hecho estos componentes se constituyen en hilos conductores para la organización del contenido.

Existe un sinnúmero de investigaciones que buscan tales hilos conductores, que reportan la utilidad de la organización del contenido a través de elementos que pertenecen al modo de actuar del profesional y no a los conocimientos.

Así pues, Gutiérrez cita a Blanco (1998), Vázquez (1999) y Yordi 
(2003) como autores que organizan los contenidos en Matemática a través de habilidades. En Física a Ortiz (2002), Falcón (2002) y Herrera (2003), quienes organizan el contenido a través del método y habilidades. A través de tareas, a García (2000), Comparan (2002) y Garza (2002). Sin embargo estos trabajos sólo contemplan la relación que existe entre un área determinada del saber y el modo de actuar del profesional, y no la relación que debe existir entre todas las áreas, las que pueden hacer variar la organización del contenido en determinados momentos, hacia uno u otro extremo de la contradicción fundamentalizacióprofesionalización.

Con igual postura de considerar los modos de actuación del profesional como los aspectos esenciales que deben regular todo el proceso de diseño de los planes y programas de estudio en todos los niveles de sistematicidad del proceso: áreas, disciplinas, asignaturas o módulos, niveles o años y temas, el Centro de Estudios de Educación Superior (CEES) Manuel F. Gran, en la Universidad de Oriente, Cuba, creó a finales de los años 90 un modelo de diseño curricular llamado Modelo de Actuación Profesional (MAP) y su correspondiente metodología curricular, que posteriormente es superado por un nuevo modelo que crean ellos mismos: el Modelo Curricular con base en las Competencias Profesionales (MCP), en correspondencia con la tendencia internacional de los últimos años de los currículos por competencias, y de diseñar un currículo fundamentalmente orientado hacia la formación integral del futuro profesional, que lo haga competente dentro del escenario social.

En el caso del MAP, los modos de actuación del profesional son llevados al diseño de la carrera, en todos sus componentes, a través del concepto de Lógica Esencial de la Profesión (LEP), como expresión didáctica de los modos de actuación del profesional. Ello significa determinar cuáles son las habilidades, los conocimientos y los valores más generales (invariante de contenido de la carrera), de los que debe apropiarse el estudiante para que, como profesional, pueda resolver de manera creativa los problemas más generales y frecuentes de su profesión.

En el caso del MCP, la profesión se lleva al plano didáctico a través de las competencias profesionales, consideradas "ejes curriculares" que atraviesan el proceso curricular y llegan a todos los niveles de sistematicidad del proceso. Las competencias profesionales sirven de enlace entre la profesión y el contenido, pues la profesión se expresa en términos de contenido a través de aquellas, las que se constituyen a partir de invariantes de habilidad, invariantes de conocimientos y valores profesionales, es decir, la invariante de contenido, que expresa la lógica o el modo que actúa el profesional.

Aunque ambos modelos aportan nuevos conceptos como la LEP, situaciones profesionales y competencias profesionales, se basan en los mismos principios del modelo cubano de perfil amplio y en su sistema de categorías. Además utilizan el mismo 
proceso de derivación vertical, desde el nivel de carrera, hasta el nivel de tema, con dichas categorías.

Los autores del artículo consideran que una limitante de ambos modelos es la de continuar asumiendo de antemano una rígida estructura organizativa de la carrera en cinco años académicos, divididos en dos semestres cada uno.

Uno de los aspectos positivos de la metodología que propone el MAP, y que lo distingue de metodologías anteriores a ella, es la precisión que logra sobre la dialéctica entre objetivo y contenido en cuanto al diseño de las asignaturas del año, ya que asocia a cada año situaciones profesionales que determinan los propósitos del año, que a influyen en el diseño de las asignaturas. Al mismo tiempo la ubicación de dichas asignaturas da precisión a los propósitos del año, obteniendo finalmente los objetivos del año. Sin embargo, no se da una explicación teórica de cómo se desarrolla la dinámica del proceso de ubicación de las asignaturas ni se proporciona un instrumento metodológico que permita ubicar con criterios sólidos y de profundas bases científicas las asignaturas de la carrera.

El MCP mantiene el logro de la precisión sobre la dialéctica objetivocontenido que plantea el MAP, y lo lleva hasta el nivel de semestre. Considera que los objetivos del semestre y sus competencias tienen que ser la integración de los objetivos de las asignaturas o módulos del semestre. Además, la ubicación de una asignatura en un semestre no puede dejar de tener en cuenta su adecuada correspondencia con el objetivo del semestre y la competencia que se alcanza como acercamiento al profesional. Plantea que el diseño del semestre debe transcurrir de forma paralela a la concepción de las asignaturas, es decir, que después de definir los propósitos más trascendentes que se deben lograr cada año, a partir de las situaciones profesionales, se debe ir definiendo la derivación de las asignaturas en los semestres según estos propósitos y la propia ubicación de las asignaturas o módulos. No obstante, sigue la carencia del fundamento teórico y su correspondiente instrumento que permita ubicar con criterios sólidos y de profundas bases científicas las asignaturas o módulos.

EI MCP no precisa los criterios que deben regir el diseño de las asignaturas o módulos, de manera que se garantice la articulación necesaria entre las asignaturas de diferentes áreas en la estructura previamente asumida de semestres para la carrera, ya que sólo plantea que la asignatura (módulo) debe ser el resultado de la derivación del invariante de contenido del área y que esta derivación puede hacerse atendiendo a diferentes criterios que dependen de los propios contenidos del área, del papel del área y las asignaturas en el programa académico, de las concepciones didácticas y curriculares que se asuman, entre otras.

Las metodologías curriculares existentes dedican mucha atención al diseño de los componentes del plan de estudio individualmente, pero descuidan la interacción de dichos compo- 
nentes y no propician que funcionen como un sistema óptima, incurriendo así en un problema de incompatibilidad práctica del sistema.

Otro factor que afecta el funcionamiento como sistema del plan de estudio, y que tiene que ver con la organización del contenido, es que la propia estructura que se da a partir de las relaciones que existen entre las partes componentes del contenido, se ve violentada al tener que cumplir una estructura organizativa de la carrera, previamente asumida, que no coincide con la primera. Esto indica la poca flexibilidad del plan de estudio, no sólo esta dada por lo anterior, sino también porque obliga al estudiante a asimilar el contenido de enseñanza en un único orden predeterminado por los expertos.

Ya Tyler (1986: 99) expresaba que cada vez es más generalizado el reconocimiento de la diferencia que existe entre una organización lógica, enfocada por quienes dominan los elementos que se van a enseñar, y una organización psicológica, que tiene que ver con el estudiante, como aparece ante sus ojos. En muchos aspectos una organización lógica que tiene un significado preciso para un experto en la materia, resulta también una organización psicológica apropiada y deviene un esquema de desarrollo con relaciones significativas para el estudiante, pero otras veces existe una diferencia categórica entre las conexiones que capta el experto en la materia y los desarrollos que resultan significativas para el propio estudiante.

Cruz y Fuentes (1998: 30) reconocen la importancia de que el estudiante construya su propio currículo en respuesta a sus expectativas y necesidades, ya que en este caso el estudiante deberá comportarse durante su proceso de formación con elevados niveles de motivación y compromiso, y buscará acceder con un gran interés a las informaciones que le permiten enfrentar y resolver los problemas que se le presentan.

La rigidez en el currículo no ayuda ni a los docentes ni a los estudiantes, por lo que debe quedar un espacio que ambos puedan ampliar u omitir, dependiendo de sus motivaciones y características reales. El no aceptar temas que el estudiante desea incorporar o eliminar afecta la motivación hacia dicha temática; de ahí que es importante colegiar las decisiones curriculares con el estudiante dándole participación en los mismos.

Por todo lo anterior, el diseño curricular debe dar como resultado un currículo en que estén en equilibrio los elementos que le dan flexibilidad y, al mismo tiempo, rigidez. Así mismo, que dicha flexibilidad, además de permitir atender a diferencias individuales en el proceso de enseñanza aprendizaje, contribuya también a la motivación y el interés del estudiante. 


\section{Conclusiones}

- La necesidad de organizar el contenido de enseñanza es una función del diseño curricular reconocida por diferentes autores, cualquiera que sea el modelo curricular a que respondan, incluso dentro de las fuentes a tener en cuenta para el diseño curricular.

- El proceso de organización del contenido es muy complejo y además no existe una única forma de organizarlo, por tanto, requiere instrumentos metodológicos adecuados que lo faciliten.

- En los años de 1970 se comenzó a aplicar métodos de la Teoría de Grafos en la organización del contenido de enseñanza, que alcanzó su madurez en los 80 , pero su uso fue cuestionado por su visión técnica y poco reflexiva, que no satisfizo las expectativas creadas. Su posterior uso hasta la actualidad fue mínimo, a pesar del desarrollo alcanzado por la tecnología computarizada.
- Las metodologías de diseño curricular existentes incurren en un problema de incompatibilidad práctica del plan de estudio como sistema, lo que afecta su funcionamiento, por asumir una estructura organizativa rígida de la carrera.

- El plan de estudio diseñado es rígido en cuanto a la organización del contenido de enseñanza que ofrece al estudiante, lo cual afecta la motivación y el interés del estudiante en el plan. Por tanto se requiere una organización del contenido de enseñanza que permita un plan de estudio que funcione adecuadamente como sistema. En este sentido, la organización del plan de estudios debe propiciar la interacción de sus componentes con flexibilidad que permita al estudiante individualizar su currículo y colaborar a que funcione mejor como sistema.

\section{Bibliografía}

Añorga, M.J. (1997). Currículo y diseño curricular. Aproximaciones metodológicas al diseño curricular. <http:// jagua.cfg.sld.cu/〜aldo/diplomado/web2/ pages/docs/modulo2/01.doc>

Bobkov, E.I. (1980). Experiencia en la elaboración de planes docentes en un CES de Ingeniería. E.I. Bobkov, D.A. Butaev. Revista La Educación Superior Contemporánea. 2(30),99-104.

Cruz, S. (1988). Modelo de actuación Profesional: una propuesta viable para el di- seño curricular de la educación superior. Silvia Cruz, Homero C. Fuentes. Santiago de Cuba: Universidad de Oriente.

Daros, W. (1993). Construcción de los conocimientos y diseño curricular de base epistemológica. Revista IRICE. (7),5-53.

Díaz Barriga, Á. (1994). Currículo y tecnología educativa. Revista tecnología y comunicación educativas. (25),3-11.

Dimova, V. (1981). La organización optima del contenido de la enseñanza. Venera 
Dimova, Dobromiv Malamov, Venelin Chalykov. Revista La Educación Superior Contemporánea. 4(36),165-177.

Documentos Rectores del Ministerio de Educación para el trabajo científico-metodológico de carácter teórico-práctico. 1976. Resolución Ministerial 597/76. Ministerio de Educación. Cuba: Editorial de Libros para la Educación.

Enríquez, E. (2003). Seis dimensiones del currículo: una interpretación sobre Posner. Revista didac. (42),36-37.

Fuentes González, H.C. (2000). Modelo Curricular con base en competencias profesionales. Santa Fe de Bogotá: [s.n.]

Gimeno Sacristán, J. (1996). El currículo: una reflexión sobre la práctica. Madrid: Ediciones Morata, S.L.

González, A.M. (2002). Los mapas conceptuales como estrategia del proceso de enseñanza aprendizaje. En Nociones de sociología, psicología y pedagogía. Ana María González Soca, Carmen Reinoso Cápiro. La Habana: Editorial Pueblo y Edulación. pp. 185-210.

Ibarra López, A.M. (2000). La teoría curricular: un modelo explicativo de sus referentes epistemológicos y de sus principales tendencias. Revista de la Universidad del Valle de Atemajac. (37), 48.

Krasnov, N.F. (1977). Sobre los trabajos de investigación científica del problema "La organización científica de la enseñanza superior" durante los años 1971-75. Revista La Educación Superior Contemporánea.2 (18):232-262.

Lamas García, J. (1984). Un procedimiento para la articulación entre asignaturas. Jorge Lamas García... [et al]. Revista Cubana de Educación Superior. 4(4), 73-96.

Lara Guerrero, J. (1997). Estrategias para un aprendizaje significativo constructivista. Revista Enseñanza. 15, 29-50.
Loredo, J. (2003). El currículo como objeto de estudio. Revista didac. (42), 11-15.

Peón García, L. (1982). Medios técnicos de computación y su aplicación al proceso docente. Revista Cubana de Educación Superior. 2(3), 57-67.

Rodríguez Palacios, A. (1985). Consideraciones teóricas metodológicas sobre el principio de la relación intermateria a través de los nexos de concepto. Revista Cubana de Educación Superior. V (1), 95-107.

Salgado Febles, J. 1979. Importancia de las relaciones intermaterias y su preparación con métodos matemáticos en el aumento de la calidad de la enseñanza. Revista Educación. IX (32), 71-98.

Siroezhin, I.M. (1975). El Plan docente de la especialidad confeccionado por la computadora electrónica. I.M. Siroezhin, N.P. Jristova. Revista la Educación Superior Contemporánea. 3(11), 23-39.

Tyler, Ralph W. (1986). Como organizar la actividad para un aprendizaje efectivo. En Principios básicos del currículo. Buenos aires: Ed. Troquel S.A. pp. 85-106.

Trista Pérez, B. (1985). Dirección en los Centros de Educación Superior. La Habana: [s.n.].

Torres Hernández, E. (1983). Elaboración de los planes y programas de estudio. Revista Cubana de Educación Superior. 3(2), 62-71.

Voltes Bou, P. (1978). La Teoría General de sistemas. Barcelona: Editorial Hispano Europea.

Zapata Castañeda, P.N. (2001). Una aplicación de la teoría de conjuntos, la lógica de proposiciones y la teoría de grafos al análisis de mapas conceptuales. T.E.D. Revista de la Facultad de Ciencia y Tecnología. 10, 79-88. 